\title{
Design for the Multi-functional Floating Garbage Disposal Device
}

\author{
Hai-Ting ZHANG ${ }^{1, a}$, Jian-Bo CAO ${ }^{1, b_{*}}$, Jian-Xia CAO ${ }^{2, b}$,Tian-Feng ZHAO ${ }^{3, b}$, \\ Zhao GAO ${ }^{1, a}$ and Han-Pin $\mathrm{LUO}^{1, a}$ \\ ${ }^{1}$ College of Engineering, Zhejiang Normal University, Jinhua, Zhejiang 321004, P.R. China \\ 2 Jinan Jindingnuo Pump Co., Ltd, Jinan, Shandong 250100, P.R. China \\ ${ }^{3}$ Library, Zhejiang Normal University, Jinhua, Zhejiang 321004, P.R. China

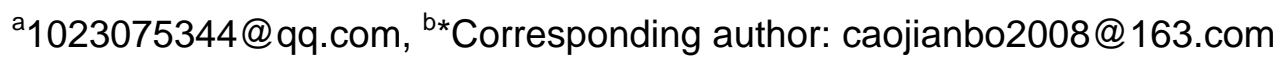

Keywords: Floating garbage, Oil-water separator, Fishing gear

\begin{abstract}
With the rapid growth of economy and population in coastal areas, floating waste has gradually become a new environmental issue. So floating junk clean-up work is exigent to be solved. Floating garbage mainly consists of floating solid waste and leakage of oil. Aiming at floating rubbish disposal, we designed a rubbish disposal device which can collect the solid waste on water and separate the oil leakage from water. This device is mainly composed of fishing gear and oil-water separator. The salvaging device is composed of a conveyor belt, poking claw, stepped shaft and supporting plate; the oil-water separator is composed of a pump, tank, and motor. The floating disposal device is convenient to use and contributes to reducing the working intensity and improving the work efficiency.
\end{abstract}

\section{Introduction}

Floating garbage has a negative impact on water environment, ecological system, economic development, human health and so forth. It mainly causes the following results: water pollution, destruction of landscape and visual pollution; damage on the function and health of water ecosystem; damage on the aquatic organisms and biodiversity; invasion of alien species attached on the garbage, which has become an international safety problem affecting the ship sailing over distance. As a result, floating waste is an invisible factor which influences the quality of coastal zone and water ecological environment [1].

The control work of floating garbage is of great significance, which is also a part of the protection of international water environment. Actions on floating junk disposal control must be taken in order to accord with the international community. Based on previous researches, we introduce a design which can be used in the daily life and deal with both floating waste and oil pollution. This design for garbage is mainly composed with four parts: a power driven device, floater collecting device, oil-water separator and the carrier device equipped with the devices above [2].

\section{Mechanical Structure Design of Floating Garbage Clearing Device}

Introduction of Device Structure. For this part, it provides a full explanation of the mechanical structure of this device, including structure of the hull, salvaging device, oil-water separating installation. At the same time, it's necessary to consider the practicability in life. There will be analysis and design on geometric parameter for each part, and then the finite element simulated analysis after completing the design.

Hull Structure Design. Hull structure is a floating carrier, which could be installed with other parts and devices. Therefore, it should take the buoyancy and installation space into account when designing the structure. According to the analysis of design structure in relevant literatures, a model structure of the hull initially came into being [3]. As shown in Fig. 1. 


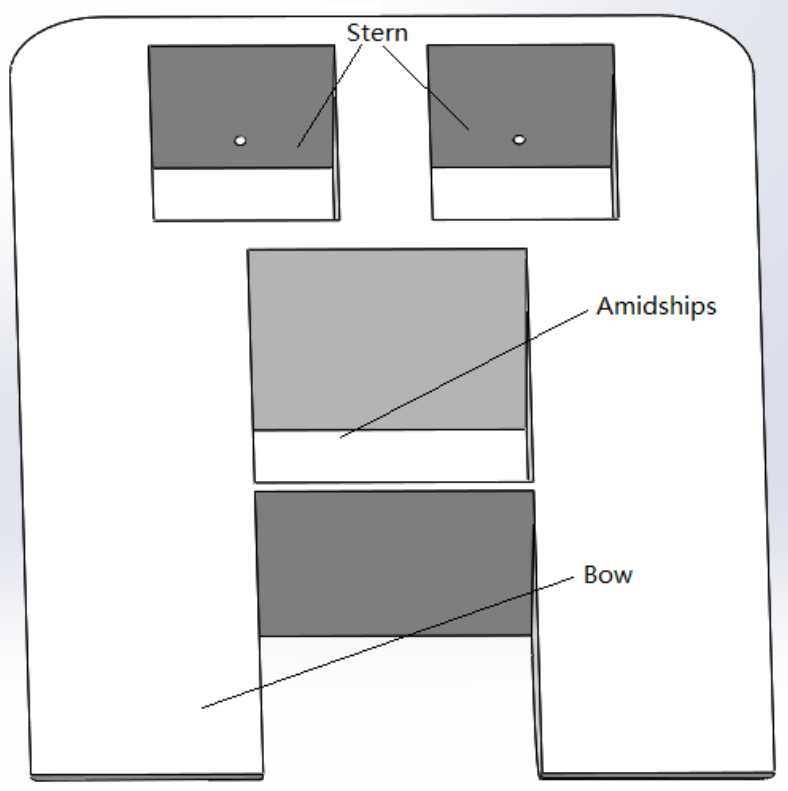

Fig. 1 The hull structure model

In the above model, the tail of hull is connected to the propeller, the middle part of the hull is used to collect garbage, and the bow to install the fishing device. According to this scheme, it's easy to calculate all the data such as two-dimensional size, vector buoyancy and so forth.

As shown in Fig. 2, the propeller is installed in the ship tail, and the thickness of the end of the hull is $50 \mathrm{~mm}$. Besides, the propeller is driven by the motor. The reserved installation space for motor is $300 \times 200 \mathrm{~mm}^{2}$, according to the motor type. The garbage is placed in the middle of the hall. With the increasing amount of the garbage in the dustbin, weight of the hull itself is also changing. In order to keep the center of gravity stable, the location of the dustbin must be consistent with the position of the center of gravity of the carrier. Only in this way can carrier keep stable and safe as the amount of garbage increases.

The buoyancy float of carriers is generated mainly by the parts on both sides, and the volume of displacement is:

$$
V=1300 \times 800 \times 200-600 \times 300 \times 200 \mathrm{~mm}^{3}=172000000 \mathrm{~mm}^{3}=0.172 \mathrm{~m}^{3}
$$

The buoyancy float of carriers is:

$$
F=\rho g v=1000 \times 9.8 \times 0.172 N=1685.6 N
$$

The $\rho$ : the density of water;

The $v$ : the volume of displacement.

Accordingly, the bearable quality of the carrier is:

$$
m=1685.6 / 9.8 \mathrm{~kg}=172 \mathrm{~kg}
$$

The quality of carriers plus accessories attached is about $100 \mathrm{~kg}$. In order to guarantee the reliabil ity and stability while working, the allowable load of garbage quality is about $40 \mathrm{~kg}$. 

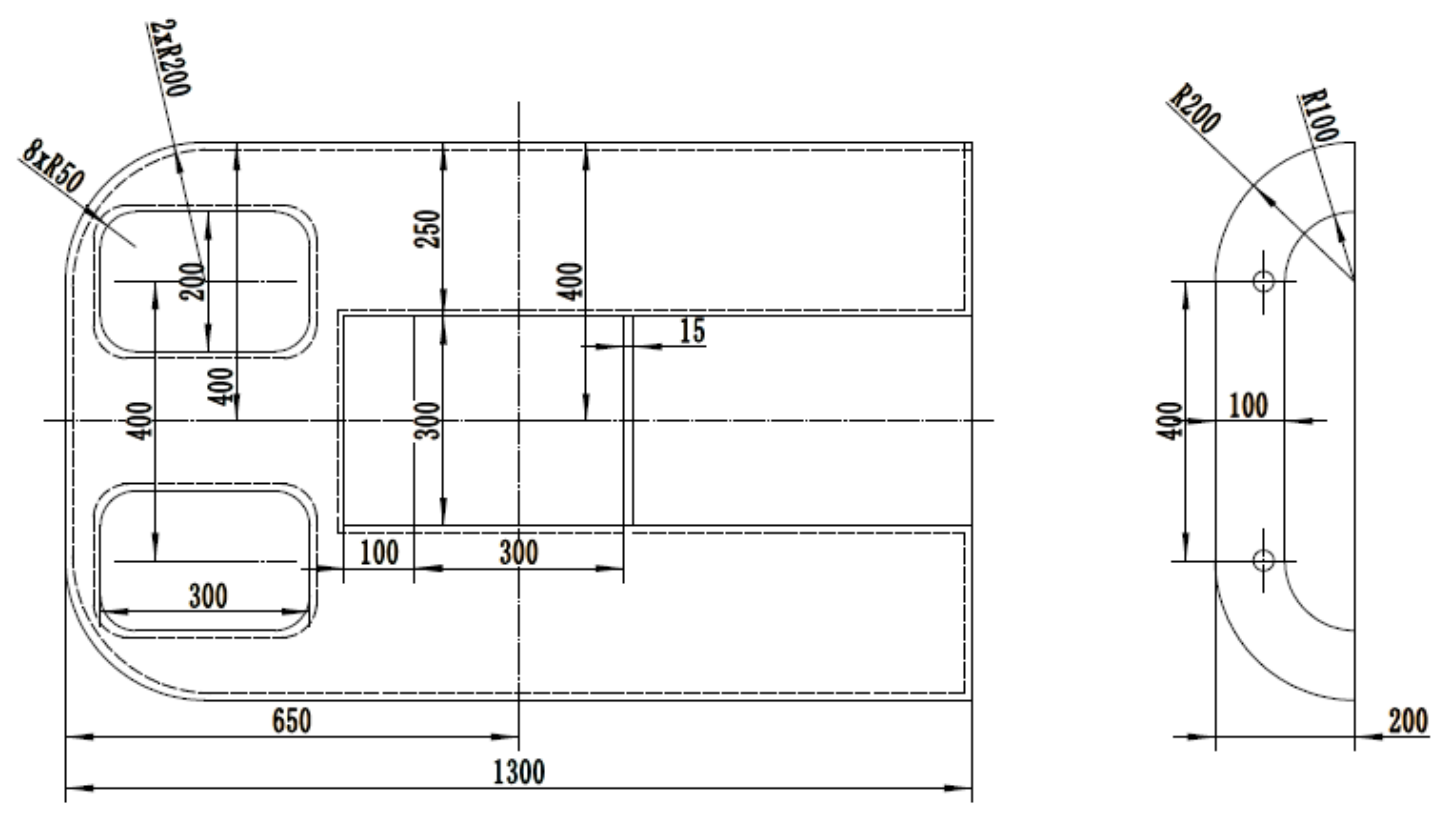

Fig. 2 The two-dimensional size

The Design of Fishing Gear. Based on the research of related literatures, this device below is designed for the fishing gear to collect floating rubbish, and then transfer the garbage floating on the water to dustbins. As shown in Fig.3, the fishing gear consists of motor conveyor belt, pusher dog , support plate and the stepped shaft [4-5].
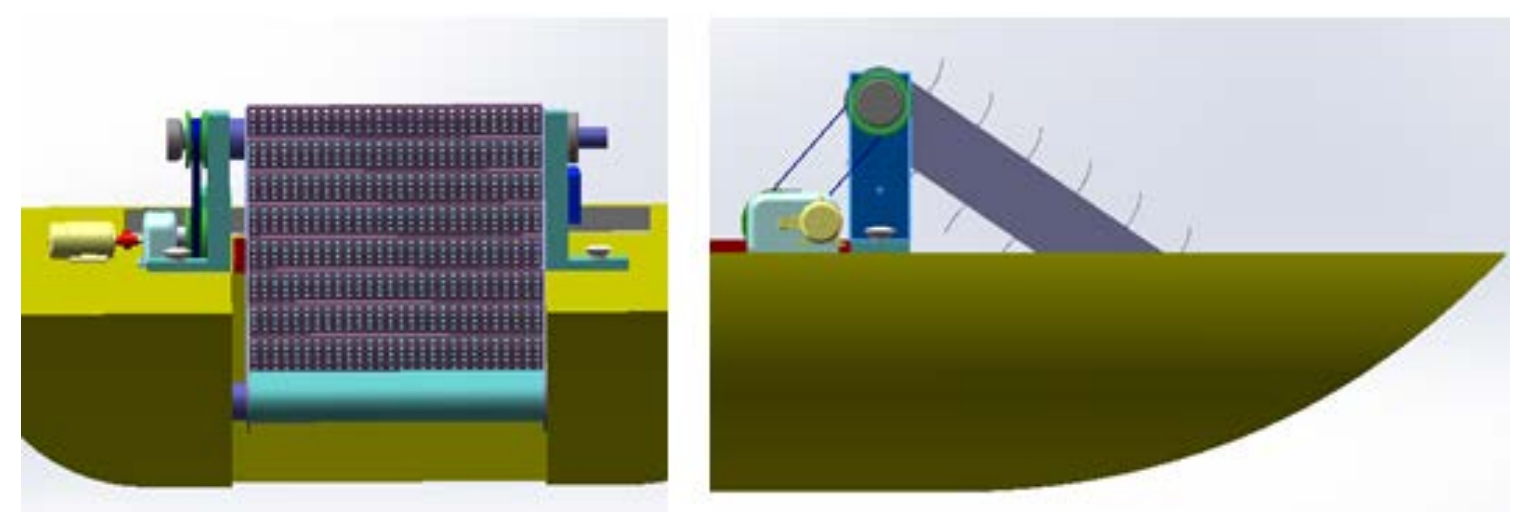

Fig. 3 3D graph of Fishing gear

The pusher dog which is installed on the conveyor that is turned by the belt pulley will move upward. The pusher dog of the conveyor will collect floating garbage. And finally the waste will drop into the dustbin because of the gravity, when moving up to the top with the conveyor. By doing so, the whole salvaging and storing work is accomplished. During the process, there are four parts to be designed: conveyor, pusher dog, pulley transmission, and design of mounting plate and stepped shaft.

The width of the conveyor belt is $300 \mathrm{~mm}$, identical with that of the dustbin. The conveyor belt will reach underwater, and the angle between the conveyor belt and the horizontal is 60 degrees.

The finger is mounted onto the conveyor belt, to collect garbage floating on water. Its three-dimensional graph is shown in Fig. 4. The different angles to get rid of waste should be taken into account. Given the situation that some of the floating waste is household garbage and branches of trees, we take the design of pusher dog stalled on the conveyor, in order to enhance the efficiency of collecting garbage. 
Fig. 4 Three-dimensional figure of pawl

According to previous experimental results, there are a variety of garbage which can be fished, including beverage bottles, plastic bags, paper cups, cloth, wood, etc. Furthermore, the passive pusher dog can be the main salvaging assistant device on aquatic plants and most of the soft thin-wall sheets. Fig. 5 is the design of the pusher dog.
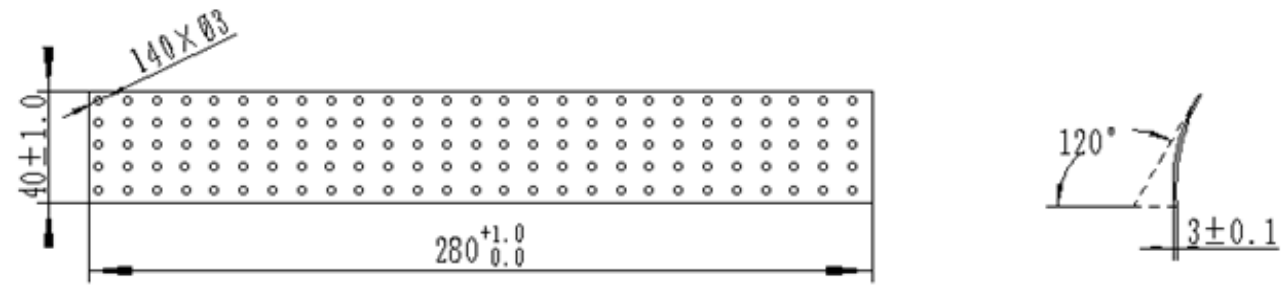

Fig. 5 The design of pawl

The Design of Oil-water Separator. Oil-water separator is designed to separate the oil and water. According to the density difference between oil and water and sedimentation theory, we can use the separator, with the diffuser cone and strainer inside, to remove both impurities and water. Both the theory and techniques of oil-water separator are mature.In this paper, we only study the main oil-water separation method and device in order to meet the design requirements, and only select practical device that is suitable for our design.

Before we choose a feasible device, the first thing is to learn about characteristics of the working environment for this device. This device will operate on the changeable surface of the water, so gravity settling method is not suitable. Besides, the separation adsorption method is also not suitable for this device for its requirement for complex equipment and precision. Overall, the centrifugation meets with our requirements. Under certain speed, the rotary centrifugation device is able to separate water and oil due to the different density between them. Currently, the oil removal of centrifugation device needs much cost and the volume is also too large. So we finally adopted the membrane separation technology for its simple structure and practicability. The whole device is shown in fig. 6 .

The working principle of this device is that when there is oil over the water,we can start the motor behind. And with the work of the pump, the oil will be sucked in. There is a fuel tank at the outlet, with a lattice beneath the tank itself. Besides, there is an absorbing film inside the tank. So when the water and oil are sucked out of the outlet, the oil will be absorbed in the film and the water will flow from the grid. Finally, the separation of oil and water is completed.
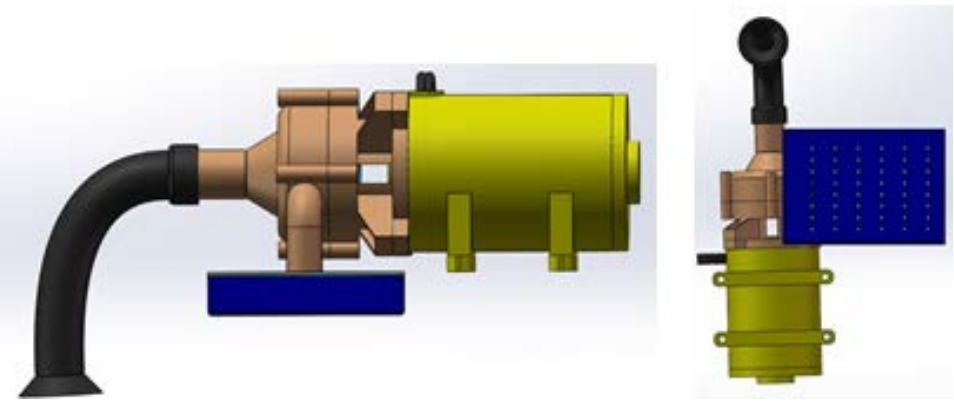

Fig. 6 Membrane separation device 


\section{Simulation Analysis of the Hull}

After carrying out the calculation of size, simulation analysis must be done to affirm whether it is practical [6-9]. In the structural design of the hull, the intermediate refuse container is the center of gravity, which actually bears almost 1000N. Fig.7 shows a displacement deformation of the hull, and fig. 8 demonstrates a strain of the hull. From the analysis of the figure, the maximum displacement deformation of hull is $1.2 \times 10^{-6} \mathrm{~mm}$, the maximum strain is $5.3 \times 10^{-8} \mathrm{~N}$. The displacement deformation and strain of the hull are in line with the actual situation, so the size of the hull design is feasible.

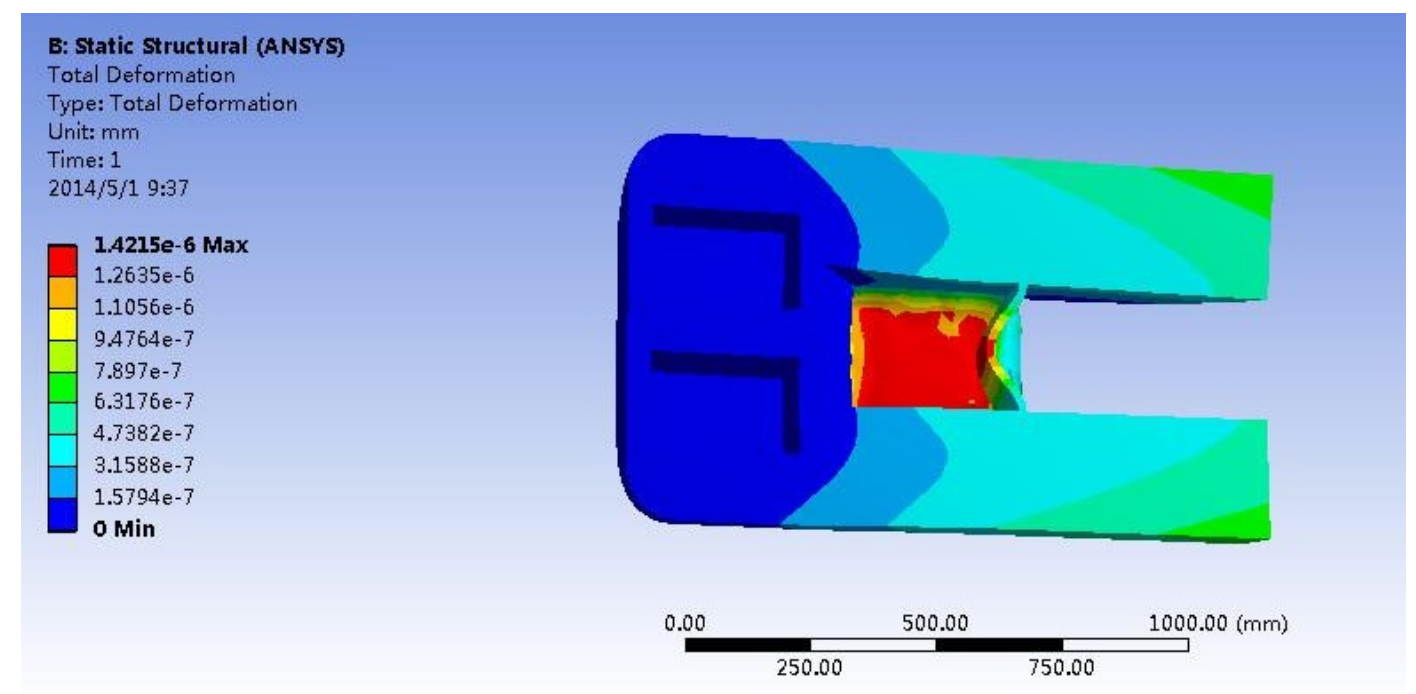

Fig. 7 Displacement deformation of the hull

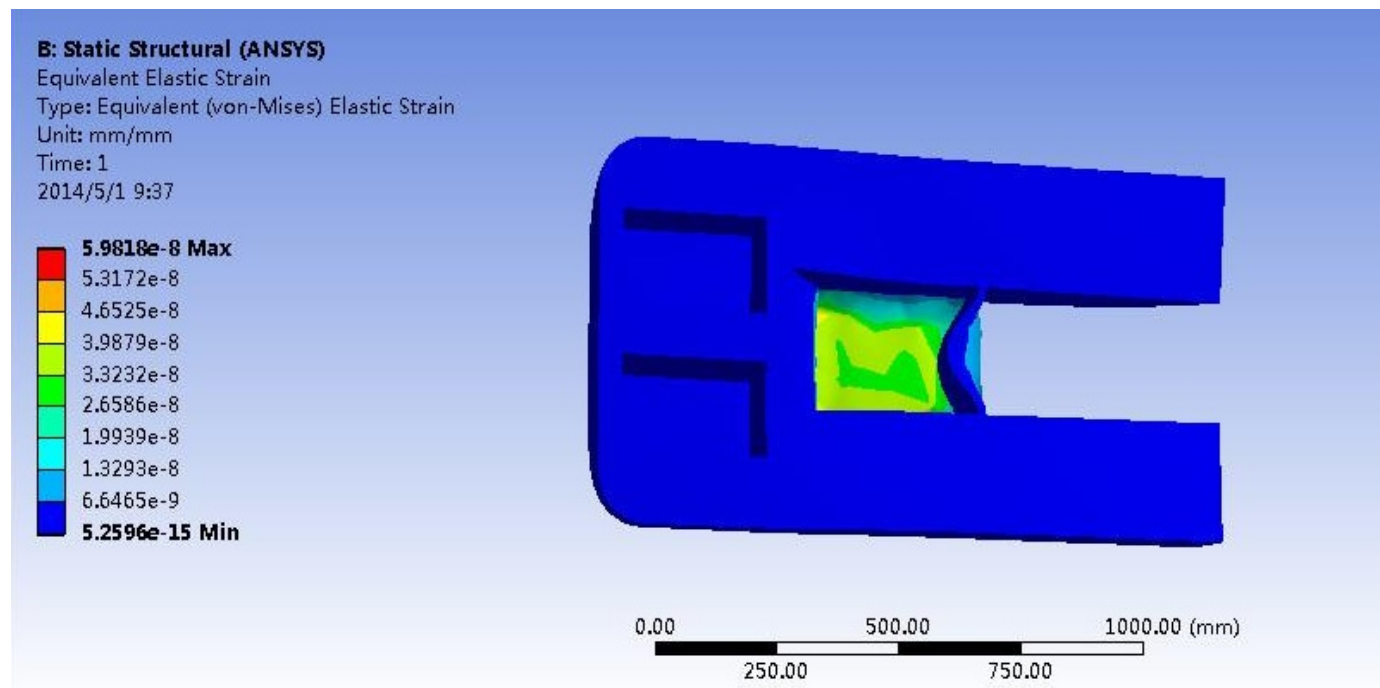

Fig. 8 Strain of the hull

\section{Conclusion}

In this paper, the fishing device is composed of a conveyor belt, poking claw, stepped shaft and support plate. Oil-water separator is composed of a pump, tank and motor. Through the power supply and the control of circuit, remote control of the device can be realized, which has a positive effect on reducing the working intensity and improving the work efficiency. In sum, this device, a combination of the garbage salvaging apparatus and oil-water separator, can contribute to the control work of floating garbage and oil leakage pollution. 


\section{Acknowledgement}

The work was supported by the National Natural Science Foundation of China (No. 51377146, 51407162), Zhejiang Provincial Natural Science Foundation of China (No. LQ13E050009), the 2013 Foundation of Education Department of Zhejiang Province of China (No. Y201329556).

\section{References}

[1] M. Zhang, J.B. Li and L.L. Chen: Water Resource and Environmental Protection, (2011) No. 3, pp. 1649-1653.

[2] Z.G. Zhao and B.Q. Shi: Remote Sensing, Environment and Transportation Engineering, (2011) No. 3, pp. 8639-9372.

[3] F.L. Gui, S.Q. Liu, L.H. Li and D. Li: Journal of Zhejiang Normal University (Natural Sciences), Vol. 38 (2015) No. 1, pp. 52-57.

[4] J.W. Chen, J.Y. Zhou, G.S. Li and M.Z. Zhou: Oceans, (2012) No. 3, pp. 1-9.

[5] Y.M. Wu, S. Zhao, Y.L. Ye, J.C. Zhu and T.X. Wu: Control Engineering and Communication Technology, (2012) No. 3, pp. 496-499.

[6] H.J. Zhang, J.B. Cao, J.J. Fang, J.W. Yu, D.D. Ma, X.M. Zhang, Y.L. Fan and X.M. Li: Science \& Technology Vision, (2015) No. 30: 81.

[7] S.D. Kim, R.T. Jung, H.T. Choi, E.J. Lee, H.G. Sung and Y.K. Lim: Oceans, (2007) No. 5: 1-5.

[8] L.X. Wang, T.F. Zhao, J.B. Cao, J.F. Shen, Y.B. Xiao and Z.X. Zhou: Key Engineering Materials, Vol. 620 (2014), pp. 335-340.

[9] J.K. Xu, J.B. Cao, J.X. Cao, F.C. Qiu, Q.H. Sun, S.J. E, H.T. Zhang and X.Z. Ding: Key Engineering Materials, Vol. 667 (2015), pp. 500-505. 